control peritoneal fluid and bowel edema. In the critically injured Urology patients, the "VAC PAC" should be more liberally used. The use of damage control in urology mainly applies to the injured ureter, where the ureter can either be ligated, or a pediatric feeding tube or ureteral stent placed up the cut ureteral edge and the stent pulled quickly through the skin. Here, definitive measures such as a Psoas hitch or Boari flap are deferred to another day when the patient is stable. Attempting definitive repairs in the critically injured patient is unwise and risks death.

\author{
Dr. Steven B. Brandes \\ Associate Professor, Division of Urologic Surgery \\ Washington University in St. Louis \\ St. Louis, Missouri, USA \\ E-mail:brandess@wudosis.wustl.edu
}

\title{
PATHOLOGY
}

\section{Does perineural invasion on prostate biopsy predict adverse prostatectomy outcomes?} Loeb S, Epstein JI, Humphreys EB, Walsh PC

James Buchanan Brady Urological Institute and the Department of Urology, Johns Hopkins Medical Institutions, Baltimore, MD, USA

BJU Int. 2009 Aug 19. [Epub ahead of print]

Objective: To determine the relationship between perineural invasion (PNI) on prostate biopsy and radical prostatectomy (RP) outcomes in a contemporary RP series, as there is conflicting evidence on the prognostic significance of PNI in prostate needle biopsy specimens.

Patients and Methods: From 2002 to 2007, 1256 men had RP by one surgeon. Multivariable logistic regression and Cox proportional hazards models were used to examine the relationship of PNI with pathological tumour features and biochemical progression, respectively, after adjusting for prostate-specific antigen level, clinical stage and biopsy Gleason score. Additional Cox models were used to examine the relationship between nervesparing and biochemical progression among men with PNI.

Results: PNI was found in 188 (15\%) patients, and was significantly associated with aggressive pathology and biochemical progression. On multivariate analysis, PNI was significantly associated with extraprostatic extension and seminal vesicle invasion $(\mathrm{P}<0.001)$. Biochemical progression occurred in $10.5 \%$ of patients with $\mathrm{PNI}$, vs $3.5 \%$ of those without PNI (unadjusted hazard ratio 3.12, 95\% confidence interval 1.77-5.52, $\mathrm{P}<0.001$ ). However, PNI was not a significant independent predictor of biochemical progression on multivariate analysis. Finally, nerve-sparing did not adversely affect biochemical progression even among men with PNI.

Conclusion: PNI is an independent risk factor for aggressive pathology features and a non-independent risk factor for biochemical progression after RP. However, bilateral nerve-sparing surgery did not compromise the oncological outcomes for patients with PNI on biopsy.

\section{Editorial Comment}

The significance of perineural invasion by carcinoma in needle prostatic biopsies is controversial $(1,2)$. Presence of perineural invasion in needle prostatic biopsies may influence the indication of resection of neurovascular bundle. Loeb's et al. study showed that perineural invasion on prostate biopsy was not a significant independent predictor of biochemical progression on multivariate analysis and nerve-sparing surgery did not adversely affect biochemical progression even among men with perineural invasion. 
In a recent study, we found that perineural invasion in needle prostatic biopsies significantly predicted prostatectomy stage $>$ pT2 in univariate $(p<0.01)$ but not in multivariate analysis $(p=0.38)$. In multivariate analysis preoperative PSA, Gleason grading and percentage of linear extent of cancer in $\mathrm{mm}$ in the needle biopsy were the significant variables predictive of $>$ pT2.

We agree with Loeb's et al. conclusion that nerve-sparing surgery does not adversely affect biochemical progression even among men with perineural invasion.

\section{References}

1. Bastacky SI, Walsh PC, Epstein JI: Relationship between perineural tumor invasion on needle biopsy and radical prostatectomy capsular penetration in clinical stage B adenocarcinoma of the prostate. Am J Surg Pathol. 1993; 17: 336-41.

2. Egan AJM, Bostwick DG: Prediction of extraprostatic extension of prostate cancer based on needle biopsy findings: Perineural invasion lacks significance on multivariate analysis. Am J Surg Pathol. 1997; 21: 1496-500.

Dr. Athanase Billis

Full-Professor of Pathology

State University of Campinas, Unicamp

Campinas, São Paulo, Brazil

E-mail: athanase@fcm.unicamp.br

\section{A pathological reassessment of organ-confined, Gleason score 6 prostatic adenocarcinomas that progress after radical prostatectomy}

Miyamoto H, Hernandez DJ, Epstein JI

The Department of Pathology, The Johns Hopkins University School of Medicine, Baltimore, MD, USA

Hum Pathol. 2009 Aug 13. [Epub ahead of print]

Prior studies of radical prostatectomies have reported a small percentage of men with biochemical progression after radical prostatectomy showing organ-confined, Gleason score 6 . One might predict that this should virtually never occur. We identified 2551 (1983-2005) radical prostatectomies coded by the urologists at our institution as pathologically organ-confined, Gleason score 6 cancer with more than 1 year of follow-up. We re-examined histopathogically the serially sectioned and completely embedded radical prostatectomy specimens of 38 men who developed biochemical recurrence defined as a single prostate-specific antigen level of $0.2 \mathrm{ng} / \mathrm{mL}$ or greater. In 27 (71\%) of 38 of cases, pathology re-review showed higher grade or stage than coded by the urologists. These included 10 cases of organ-confined with Gleason pattern 4 as either the primary or secondary pattern; 9 cases of organ-confined, Gleason score 6 with tertiary pattern 4 (in 4 cases, tertiary pattern 4 was described in the initial pathology report); 5 cases of Gleason score 7 plus extraprostatic extension; 1 case of Gleason score 6 with focal extraprostatic extension; and 2 cases with positive margins due to intraprostatic incision (listed in the initial pathology report). The remaining 11 cases were true organ-confined, Gleason score 6 tumors, but none of the patients developed systemic disease. Most prior reports of organ-confined, Gleason score 6 with progression are undergraded (upgrading with revision of Gleason system), understaged (difficulty recognizing focal extraprostatic extension), or suffer from situations with ambiguous staging (intraprostatic incision) or grading (tertiary pattern 4 or $2+4=6$ ). Even for the rare true organ-confined, Gleason score 6 (no pattern 4 ) tumor with supposed biochemical progression, some may be false-positive progression based on low post-radical prostatectomy prostate-specific antigen levels and minute tumors that seem highly improbable to progress. With accurate pathologic evaluation, men with organ-confined, Gleason score 6 (no pattern 4) prostate cancer can be told that their risk of progression is very rare $(0.4 \%)$. 